\section{MS35-04 Computational dehydration of an organic hydrate using molecular dynamics simulations}

Anders S. Larsen ${ }^{1}$, Jacco van de Streek ${ }^{1}$, Jukka Rantanen ${ }^{1}$, Kristoffer E. Johansson ${ }^{1}$

\section{Department of Pharmacy, University of Copenhagen}

email: anders.larsen@sund.ku.dk

Organic crystal structures can dehydrate to an anhydrous product, which might be completely different on important parameters such as solubility and stability. Our goal is to computationally investigate dehydration of these organic crystals at the molecular level.

Our method is based on classical molecular dynamics (1), which is able to calculate the time evolution of a crystal structure. The energy of the system is calculated with the force field COMPASS (2). The method for dehydrating the crystal is to remove the water molecules periodically during the simulation, this has proven a quite effective way to force the crystal into an anhydrous state. The time interval between the removal of the water molecules is 10 picoseconds and 2-5 are removed at a time. The systems we have investigated are prednisolone (3) and ampicillin trihydrate (4). In the bulk simulations with Prednisolone, water molecules showed great mobility in the water channels at $300 \mathrm{~K}$. This is a result of the hydrophobic nature of the channels and it confirms the non-stoichiometric nature of the Prednisolone crystal structure. For ampicillin trihydrate the water molecules did not diffuse through the crystal or along the channels, instead the hydrogen bonding network stayed largely intact at $300 \mathrm{~K}$, with the hydrogen bonding pattern extending along the channel. Although thermal motion might move the water molecule far from its equilibrium position and thus weakening the hydrogen bond, it will always return to its original position.

Water molecules are periodically removed with the previously described protocol which results in a severely disordered dehydration intermediate. As more water molecules are removed the disorder decreases. After all the water molecules are gone the crystal transform into a new anhydrate structure. Molecular dynamics is capable of describing the qualitative aspects of hydrates and shows promise for investigation of dehydration at the molecular level.

\section{References}

1. A. Nemkevich, H. Bürgi, M. A. Spackman and B. Corry, Phys. Phys. Chem. 2010; 12: 14916-14929.

2. H. Sun, J. Phys. Chem. B 1998; 102: 7338-7364.

3. S. R. Byrn, P. A. Sutton, B. Tobias, J. Frye and P. Main, J. Am. Chem. Soc. 1988; 110: 1609-1614

4. J. C. Burley, J. van de Streek and P. W. Stephens. Acta Cryst 2006; E62: o797-0799.

Keywords: Molecular Dynamics, Dehydration
MS35-O5 The application of tailor-made force fields and molecular dynamics for NMR crystallography

Xiaozhou $\mathrm{Li}^{1}$, Marcus A. Neumann ${ }^{2}$, Jacco van de Streek ${ }^{1}$

1. Department of Pharmacy, University of Copenhagen, Universitetsparken 2, DK-2100 Copenhagen, Denmark

2. Avant-garde Materials Simulation $\mathrm{GmbH}$,

Rosa-Luxemburg-Strasse 14, D-79100 Freiburg, Germany

email: xiaozhou.li@sund.ku.dk

The NMR crystallography method, which utilises solid-state NMR (SS-NMR) spectroscopy, possibly in combination with powder X-ray diffraction and $a b$ initio chemical shift calculations, is becoming attractive in the elucidation of the structural and dynamic aspects of molecular crystals. ${ }^{[1]}$ SS-NMR experiments are usually conducted at ambient temperature, and represent a time and space average. $A b$ initio SS-NMR calculations, however, are usually based on static density functional theory (DFT) calculations at zero Kelvin, leading to discrepancies between experimental and calculated chemical shifts.

For in silico calculations, the thermal motion of molecular crystals can be introduced by molecular dynamics (MD) if a force field that can properly describe the energy potential of the system of interest is available. Traditional force fields, which are generally not transferable for a wide spectrum of systems, may not represent the correct potential or configuration of a molecular crystal. ${ }^{[2]}$ Tailor-made force fields (TMFFs) are an option to overcome such a limitation: the force fields are parameterised against DFT reference data for individual molecules. ${ }^{[3]}$ Dispersion-corrected DFT (DFT-D) is well-known for its accuracy and transferability for the reproduction of molecular crystals. ${ }^{[4]}$

We will present a computational study, which aims to evaluate the performance of a tailor-made force field for the effects of thermal motion on $a b$ initio chemical shift calculations, by comparing the chemical shifts calculated for crystal structure candidates obtained from crystal structure prediction using three different approaches: static DFT-D energy minimisations, motional averaging with an existing benchmark (the COMPASS force field) ${ }^{[5}$ and motional averaging with the TMFF. The crystal structure of cocaine free base will be used as an example.

\section{References}

[1] R. K. Harris et al., NMR crystallography. (2009) John Wiley \& Sons, Chichester.

[2] A. Nemkevich et al., Phys. Chem. Chem. Phys., (2010) 12, 14916-14929.

[3] M. A. Neumann, J. Phys. Chem. B, (2008) 32, 9810-9829.

[4] J. van de Streek and M. A. Neumann, Acta Cryst., (2010) B66, 544-558.

[5] H. Sun, J. Phys. Chem. B (1998) 102, 7338-7364.

Keywords: Molecular dynamics, Tailor-made force field, Density functional theory, NMR crystallography 